\title{
The Test of Attenuation Effect of Consensus Information on In-group Attributional Favoritsm
}

\author{
Zhaoyuan Liu ${ }^{1, \mathrm{a}}$, Weijun Ma ${ }^{1, \mathrm{~b}}$, Yating Zhang ${ }^{1, \mathrm{c}}$ \\ ${ }^{1}$ The School of Psychology and Cognitive Science, East China Normal University,Putuo, Shanghai, China \\ amm12615@126.com \\ bwjma@psy.ecnu.edu.cn \\ c820475361@qq.com
}

\begin{abstract}
Intergroup Attributional Bias is an in-group-favoring and outgroup-derogating phenomenon existing in attribution. It is one kind of representation of irrational attribution arisen from the self-enhancement motivation in group level. This study discussed the influence of consensus information on Intergroup Attributional Bias. In Pre-Experiments, the subjects read and imaged some situations that contained good or bad behavior or outcome of the members of their in-group or out-group, and relevant consensus information which extents were manipulated, and made attributions in 4 causal dimensions. The results showed that high/low consensus was attributed to external environmental cause/internal dispositional cause respectively, and Intergroup Attributional Bias disappeared. The Formal Experiments discussed whether consensus information is given or not influence in-group-favoring Attributional tendency. The results indicated that in-group-favoring Attributional tendency was showed vividly when consensus information wasn't given. Nevertheless, in the condition that consensus information was given, as the results of rational attribution, high/low consensus was attributed to external environmental cause/internal dispositional cause, and in-group-favoring Attributional tendency disappeared.

In two experiments of this study, consensus information showed significant interference effect for Intergroup Attributional Bias. It is the main contribution of this study. The results of this study are discussed from the viewpoints of rational attribution and self-categorization.
\end{abstract}

Keywords: Consensus Information, Intergroup Attributional Bias, Rational Attribution, Group Salience,

Self-categorization

\section{一致性信息对内群偏祖归因的削弱效果验证}

\author{
刘昭远 $1, \mathrm{a}$ 马伟军 $1, \mathrm{~b}$ 张雅婷 $1, \mathrm{c}$ \\ 1 华东师范大学心理与认知科学学院, 普陀, 上海, 中国 \\ amm12615@126.com \\ bwjma@psy.ecnu.edu.cn \\ c820475361@qq.com
}

摘要

群际归因偏好是在归因中偏祖内群、贬低外群的现象，是由自我提高动机在群体层面表现出来的一种非理性归 因。通过两个实验检验了当人们被给予一致性信息时，是否其能通过促进理性归因并产生去类化而减弱群际归 因偏好。预实验操作一致性的程度高低, 内外群, 行为与结果的积极消极; 要求被试 4 个维度进行归因, 发现 被试对一致性信息可显著促进理性归因，且群际归因偏好表现不显著。正式实验探讨了一致性信息的有无对内 群偏袒归因倾向的影响。结果表明，无一致性信息时内群偏袒归因倾向表现显著; 而给予一致性信息时（一致 性高、低、不明确）理性归因表现显著, 内群偏袒归因倾向消失。研究说明一致性信息对群际归因偏好有显著 的干扰作用，最后从理性归因及自我归类的角度进行了探讨

关键词：一致性信息，群际归因偏好，理性归因，群体显著性，自我归类 


\section{1.前言}

人们在对事件进行归因中主要可区分为两种情 况。前者为对事件相关信息进行合乎逻辑的分析处理 而产生的归因, 是一种理性归因; 后者主要指人们的 动机、偏见、所处角度等对归因造成的影响而产生的 归因偏差, 是一种非理性归因 ${ }^{[6,11]}$ 。前者的研究产生 了如凯利的三度模型等的归因模型 ${ }^{[10]}$; 后者的研究则 主要体现在自我提高动机 (Self-enhancement motivation)对归因产生的影响。自我提高动机表现在 对自我相关的归因中则产生利已归因偏好 ${ }^{[2]}$; 表现在 对所属群体的归因中则表现出群际归因偏好 ${ }^{[8,12]}$ 。

群际归因偏好(Intergroup Attributional Bias)指在 归因中偏祖内群、贬低外群的现象; 按照 Pettigrew ${ }^{[16] 、}$ Hewstone ${ }^{[8]} 、 \mathrm{Ma}^{[12]}$ 的观点, 群际归因偏好表现于人们 在归因中容易将内群成员好的行为与结果(或成功)归 于其能力、努力、责任等内因, 将其不好的行为与结 果(或失败)归于其运气、任务难度等外因; 但对于外 群成员的行为与结果, 内群成员则进行相反的归因 ${ }^{[1,}$ $5,7,17,19,21]$ 。从动机角度来看, 产生群际归因偏好的本 质为自我提高动机, 人们对内群进行自我归类(selfcategorization)、认同致使自我提高动机扩展至内群层 面, 并且在对内外群成员的行为与结果的归因中表现 出来而产生群际归因偏好。认知角度的解释认为, 在 对群体成员的行为进行归因时人们容易将其与对该 群体的刻板印象和偏见等联系起来, 并以此为根据进 行归因 ${ }^{[8]}$ 。

群际归因偏好作为一种非理性归因, 如果给予能 促进其理性归因的线索或背景情报, 发生事件的原因 被合乎逻辑地分析处理, 将导致内外群归因差异的显 著性、及对内群的自我归类意识降低。内群的自我归 类的非显著化(或者是降低内外群的区分的显现化)、 即去类化(decategorization)发生时 ${ }^{[3,5,18,20]}$ 偏袒内群的 倾向减弱, 群际归因偏好将得到抑制。而其他研究表 明, 一致性、一贯性、区别性、偶然性等的 4 种共变 信息分别在原因的内外、稳定性、普遍性、可控制性 维度产生显著影响, 是引起理性归因的重要线索 ${ }^{[13,20]}$ 本研究选取一致性信息作为引起理性归因的线索及 背景情报, 比较给予与不给予一致性信息时群际归因 偏好的差异。可以预测只给予关于内外群成员的行为 或结果(如成功与失败)的信息、而不给予与之相关联 的一致性信息时, 内外群的显著性及对内群的自我归 类意识显著, 群际归因偏好表现显著。但两者同时给 予时, 对于一致性信息进行理性归因的同时, 产生去 类化以致内外群的显著性降低, 群际归因偏好收到削 弱。因此, 给予一致性信息时比不给予时群际归因偏 好减弱, 一致性信息的有无对群际归因偏好产生显著 影响。到目前为止, 认为一致性信息对利已归因偏好 没有显著的削弱效果, 并认为利已归因偏好是一种稳 固的归因偏好，给予一种共变信息时对其并无显著的 干扰作用 ${ }^{[6,14]}$ 。但未发现有研究探讨一致性信息对群 际归因偏好是否有削弱效果。

本研究有三个目的。第一, 本研究试图验证一致
性信息对内群偏袒归因的削弱效果。在预实验中, 探 讨给予一致性信息时是否表现出群际归因偏好。预测 在给予一致性信息时，对内外群成员的归因中群际归 因偏好减弱或消失。正式实验则探讨一致性信息的有 无对内群偏袒归因倾向的影响。预测给予一致性信息 时比不给予时理性归因显著, 但内群偏袒归因倾向减 弱。第二, 探究一致性信息将削弱群际归因偏好的哪 些层面。预测一致性高/低时人们的内外归因偏好将 受到影响。 $\mathrm{Ma}^{[13]}$ 的结果表明一致性高/低时契合法与 差异法分别起作用分别被归于外/内因。第三, 检验群 际归因偏好是否表现在 4 个原因维度。预测群际归因 偏好不仅可表现在原因的内外维度, 也可表现在稳定 性、普遍性、可控制性维度 ${ }^{[3,9]}$ 。

本研究主要探讨给予一致性信息时是否表现出 群际归因偏好, 提出并验证以下两个假设。; 假设 1: 内群偏袒归因倾向表现在 4 个原因维度, 即内群成员 的成功比失败被归于更为内在的、稳定的、普遍的、 可控的原因; 假设 2: 无一致性信息时比有时内群偏 袒归因倾向表现更为显著。

\section{2. 预实验}

\section{1. 被试}

广东省梅州市的客家人大学生 51 人(男性 25 人, 女性 26 人)。在广东省梅州市, 本地人为客家人, 也 有一些作为外来人口的外省民工。对于客家人大学生 来说, “客家人”为内群、“外省民工”为外群。实验前, 主试首先询问被试是否是客家人, 并且说明属于客家 人的大学生才能完成本问卷。

\section{2. 实验设计}

本实验参照 $\mathrm{Ma}^{[13]}$ 中给予情景的方式呈现刺激材 料。本实验的实验设计为 2 (一致性程度: 高 vs. 低) $\times 2$ (行为或结果的好坏：好 vs. 不好 $) \times 2$ (情景：面试 vs. 公共汽车) $\times 2$ (主人公所属群体：客家人 vs. 外省民 工)的 4 因素实验设计。主人公的所属群体为被试间 变量, 其余为被试内变量。

\section{3. 实验设计}

本实验参照 $\mathrm{Ma}^{[13]}$ 中给予情景的方式呈现刺激材 料。本实验的实验设计为 2 (一致性程度: 高 vs. 低) $\times 2$ (行为或结果的好坏：好 vs. 不好) $\times 2$ (情景：面试 vs. 公共汽车) $\times 2$ (主人公所属群体: 客家人 vs. 外省民 工)的 4 因素实验设计。主人公的所属群体为被试间 变量, 其余为被试内变量。

\section{4. 实验流程}

主人公所属群体的操作中, 客家人条件中在各情 景中对被试提示的主人公为“客家人小李”, 外省民工 条件中对被试提示的主人公为“外省民工小李”。情景 
的操作中，给予了被试面试与公共汽车两个情景。各 自的情景中，给予了被试关于主人公的行为或结果的 信息。“面试”情景中，设定了主人公参加某个化学公 司招聘面试的情景。在此情景的结果好坏的操作中, 主人公面试合格为“好”结果、不合格为“不好”结果。 “公共汽车”情景中, 设定了主人公乘坐公共汽车的情 景。在此情景中的行为好坏的操作中, 主人公给背着 重行李的乘客让座为“好”行为、未让座为“不好”行为。 一致性程度的操作中, 两个情景中主人公的行为或结 果与该状况中别人的行为或结果一致时为“一致性 高”、不同时为“一致性低”。

对由 3 个被试内变量组合形成的 8 个故事情节, 顺序采用拉丁方平衡。对于所有的故事情节, 要求被 试回答主人公的行为或结果是由什么原因产生的、并 在 4 个原因维度(内外、稳定性、普遍性、可控制性维 度)中回答行为或结果产生的原因(7 段尺度)。

\section{5. 实验结果}

\subsection{1, 数据处理}

通过 IBM SPSS Statistics 22.0 软件进行数据处 理, 在 4 个原因维度, 将各个条件中两个情景的得 分进行平均后作为因变量, 进行 2 (一致性程 度) $\times 2$ (主人公所属群体) $\times 2$ (行为与结果的好坏) 的 3 自变量混合实验设计方差分析。结果如下所示。

\subsection{2, 内外维度}

在内归因/外归因维度上一致性程度的主效应显 著, $F(1,48)=17.93, p<0.001$, 即与一致性高时 $(M=4.54)$ 相比一致性低时 $(M=5.20)$ 被归于较为内在的 原因。

一致性程度 $\times$ 所属群体的 2 因素交互作用有显著 倾向, $F(1,48)=3.56, p<0.10$ 。进一步检验的结果表 明, 主人公是外省民工时一致性程度的单纯主效果 显著 $(p<0.001)$, 即与一致性高时 $(M=4.40)$ 相比低时 $(M=5.35)$ 被归于较为内在的原因。但主人公是客家 人时一致性程度的单纯主效应不显著。

行为与结果的好坏的主效应显著, $F(1$, $48)=11.98, p<0.01$, 与不好的行为或结果 $(M=4.67)$ 相 比较, 好的行为或结果 $(M=5.07)$ 被归于更为内在的 原因。即在原因的内外维度表现出同程度的偏祖内 群与外群的归因倾向。

\subsection{3，稳定性维度}

稳定性维度上, 一致性程度的主效应显著, $F(1$, $48)=7.57, p<0.01$ 。即与一致性高时 $(M=3.85)$ 相比较, 低时 $(M=4.22)$ 被归于较稳定原因。

行为与结果的好坏的主效应显著, $F(1,48)=18.88$, $p<0.001$ 。即与不好的行为与结果 $(M=3.74)$ 相比, 好
的行为与结果 $(M=4.34)$ 被归于较稳定原因, 在原因的 稳定性维度表现出同程度的偏袒内群与外群的归因 倾向。

一致性程度×好坏的 2 因素交互作用显著, $F(1$, $48)=4.46, p<0.05$ 。进一步检验的结果表明, 一致性 高时 $(p<0.05)$ 、一致性低时 $(p<0.001)$ 行为与结果的 好坏的单纯主效应均显著。即与不好的行为与结果相 比, 好的行为与结果被归于较稳定原因, 此倾向特别 在一致性低时更为显著。

\subsection{4, 普遍性维度}

在普遍性维度上, 一致性程度的主效应显著, $F$ $(1,48)=5.28, p<0.05$ 。即与一致性高时 $(M=4.55)$ 相比, 一致性低时 $(M=4.83)$ 被归于较普遍原因。

行为与结果的好坏的主效应显著, $F(1,48)=8.83$, $p<0.01$ 。即与不好的行为或结果 $(M=4.54)$ 相比, 好的 行为或结果 $(M=4.84)$ 被归于较普遍原因, 在原因的普 遍性维度表现出同程度的偏祖内群与外群的归因倾 向。

\subsection{5, 可控制性维度}

在可控制性维度上, 一致性程度的主效应有显著 倾向, $F(1,48)=3.21, p<0.10$, 即与一致性高时 $(M=4.74)$ 相比较, 低时 $(M=4.95)$ 有被归于可控原因的 倾向。

行为与结果的好坏的主效应显著, $F(1,48)=12.18$, $p<0.01$,与不好的行为与结果 $(M=4.62)$ 相比较, 好的 行为与结果 $(M=5.08)$ 被归于可控原因, 即在原因的可 控制性维度表现出同程度的偏袒内群与外群的归因 倾向。

\section{6. 讨论}

首先，在各原因维度一致性程度的主效应均显著。 说明一致性信息使得被试归因于更内在、稳定、普遍、 可控的原因上; 一致性信息的确可影响被试的归因倾 向; 同时在原因的 4 个维度行为与结果的好坏的主效 应均显著, 即主人公的成功比失败均被归于更为内在 的、稳定的、普遍的、可控的原因。其次, 本实验结 果并不能验证客家大学生的群际归因偏好, 由于本实 验中只控制一致性信息的高低，因而有理由怀疑是一 致性信息的理性归因干扰了群际归因偏好。为了对此 进行进一步探讨，在正式实验中设置了一致性信息有 与无的情景, 比较其对内群偏祖归因倾向的影响。

\section{3. 正式实验}

\section{1. 被试}

广东省梅州市的客家人大学生 35 人(男性 16 名, 女性 16 名, 性别不明者 3 名)。 


\section{2. 实验设计}

正式实验采用与预实验同样的方法, 给予被试故 事情境。本实验设计为 4(一致性程度: 无 vs. 不明确 vs. 高 vs. 低) $\times 2$ (主人公面试结果: 成功 vs. 失败)的 2 被 试内实验设计。被试属于“客家人”群体, 本实验的故 事情景中的主人公“客家人小李”对被试来说是代表 内群体成员的刺激人物。

\section{3. 实验流程}

本实验中设定了主人公参加某个化学公司的招 聘面试的情景。一致性程度的操作中, 主人公在这个 面试中的结果与其他全部面试者一样时为“一致性高” 条件，相反为“一致性低”条件。“一致性不明确”条件 中, 别的面试者中, 一些人获得与此人同样的结果、 一些人获得相反的结果。“无一致性信息”条件中, 完 全没有给予与一致性信息相关联的信息。面试结果的 操作中, 主人公在这个面试中合格为“成功”、不合格 为“失败”。

对两个被试内变量进行组合形成 8 个故事情节, 为减少误差颠倒其提示顺序, 从而获得两套问卷, 每 套问卷均含有 8 个故事情节。从中随机抽取其中一套 让被试完成。对每个故事情节, 通过 4 个原因维度 $(7$ 段尺度)测量被试对主人公面试结果的归因。

\section{4. 结果}

\section{4 .1 , 数据处理}

通过 IBM SPSS Statistics 22.0 软件进行数据处理, 各原因维度的得分作为因变量, 进行 4(一致性信息程 度) $\times 2$ (内群成员面试结果)的 2 被试内自变量方差分 析。结果如下所示(表 1)。

\subsection{2, 内外维度}

一致性程度的主效应显著, $F(3,99)=13.20, p<$ 0.001。用 LSD 法进行多重比较后发现，与其他 3 个 条件相比一致性高时被归于较为外在的原因。面试结 果的主效应有显著倾向, $F(1,33)=3.75, p<0.10$, 即 内群成员的成功比失败有被归于更为内在的原因的 倾向，表现出内群偏祖归因倾向。

一致性程度 $\times$ 面试结果的交互作用显著, $F(3$, $99)=2.84, p<0.05$ 。简单效应检验结果表明：内群成 员的成功比失败被归于较为内在的原因。但在一致性 高、低及不明确的 3 个条件中, 内群成员面试结果的 单纯主效应不显著。因此, 无一致性信息条件中表现 出内群偏袒归因倾向, 但有一致性信息的 3 个条件中 没有表现出内群偏袒归因倾向。即与有一致性信息的 3 个条件相比, 无一致性信息时内群偏袒归因倾向表 现显著。

\subsection{3，稳定性维度}

一致性程度的主效应显著, $F(3,96)=6.81, p<$ 0.001 。运用 LSD 法进行多重比较后发现, 与一致性 不明确及高时相比较一致性低时、与一致性高时相比 无一致性信息时, 被归于较为稳定的原因。面试结果 的主效应显著, $F(1,32)=5.50, p<.05$ 。即内群成员 的成功比失败被归于更为稳定的原因, 在稳定性维度 表现出内群偏袒归因倾向。但一致性程度 $\times$ 面试结果 的交互作用不显著。

\subsection{4, 普遍性维度}

一致性程度的主效应显著, $F(3,99)=8.54, p<$ 0.001 。运用 LSD 法进行多重比较后发现, 与其它 3 条件相比, 一致性高时被归于较为特殊的原因。面试 结果的主效应显著, $F(1.33)=5.04, p<0.05$ 。即成功 比失败被归于内群成员较为普遍的原因，在普遍性维 度表现出内群偏袒归因倾向。一致性程度 $\times$ 面试结果 的交互作用不显著。

\subsection{5, 可控制性维度}

一致性程度的主效应有显著倾向, $F(3,96)=2.51$, $p<0.10$ 。运用 LSD 法进行多重比较后发现, 一致性 低与不明确时比高时被归于较为可控的原因。面试结 果的主效应显著, $F(1,32)=23.19, p<0.001$ 。即内群 成员的成功比失败被归于内群成员的更为可控的原 因，在可控制性维度表现出内群偏袒归因倾向。

一致性程度 $\times$ 面试结果的交互作用显著, $F(3,96)$ $=3.89, p<0.05$ 。进一步检验的结果表明, 一致性低时 $(p<0.001)$ 、无时 $(p<0.01)$ 、不明确时 $(p<0.05)$ 面试 结果的单纯主效应显著。即这 3 个条件中成功比失败 被归于内群成员的更为可控的原因。但一致性高时面 试结果的单纯主效应不显著。因此，在原因的可控制 性维度, 除了一致性高外的 3 个条件中表现出内群偏 袒归因倾向。

\section{表 1 在各个原因维度对于内群成员的面试结果的 归因得分 $M(S D), N=35$}

\begin{tabular}{cccc}
\hline $\begin{array}{c}\text { 原因维度与 } \\
\text { 一致性程度 }\end{array}$ & 面试结果 & \\
\hline 内外维度 & & 失败 & 平均值 \\
无 & $5.53(1.54)_{1}$ & $4.59(1.46)_{2}$ & $5.06(1.19)_{\mathrm{b}}$ \\
不明确 & $5.24(1.44)$ & $5.12(1.49)$ & $5.18(1.24)_{\mathrm{b}}$ \\
高 & $3.68(1.92)$ & $3.79(1.95)$ & $3.74(1.75)_{\mathrm{a}}$ \\
低 & $5.47(1.88)$ & $5.32(2.11)$ & $5.40(1.89)_{\mathrm{b}}$ \\
平均值 & $4.98(1.19)_{l}$ & $4.71(1.24)_{2}$ & \\
\hline 稳定性维度 & & & \\
无 & $4.09(1.47)$ & $3.18(1.55)$ & $3.64(1.21)_{\mathrm{c}}$ \\
\hline
\end{tabular}




\begin{tabular}{|c|c|c|c|}
\hline 不明确 & $3.52(1.37)$ & $3.21(1.54)$ & $3.36(1.15) \mathrm{a}$ \\
\hline 高 & $3.33(1.55)$ & $2.88(1.43)$ & $3.11(1.09)_{\mathrm{ac}}$ \\
\hline 低 & $4.30(1.55)$ & $3.76(1.85)$ & $4.03(1.27)_{b}^{b}$ \\
\hline 平均值 & $3.81(0.99)_{1}$ & $3.26(1.26)_{2}$ & \\
\hline \multicolumn{4}{|c|}{ 普遍性维度 } \\
\hline 无 & $4.21(1.61)$ & $3.82(1.47)$ & $4.01(1.23)_{b}$ \\
\hline 不明确 & $4.35(1.65)$ & $3.85(1.64)$ & $4.10(1.28)_{b}^{b}$ \\
\hline 高 & $3.32(1.59)$ & $3.12(1.37)$ & $3.22(1.15)_{\mathrm{a}}$ \\
\hline 低 & $4.65(1.70)$ & $4.24(1.92)$ & $4.44(1.50)_{b}$ \\
\hline 平均值 & $4.13(1.13)_{1}$ & $3.76(0.99)_{2}$ & \\
\hline \multicolumn{4}{|c|}{ 可控制性维度 } \\
\hline 无 & $4.64(1.58)_{1}$ & $3.76(1.48)_{2}$ & $4.20(1.15)$ \\
\hline 不明确 & $4.73(1.35)_{1}$ & $3.91(1.53)_{2}$ & $4.32(1.18)_{b}$ \\
\hline 高 & $3.94(1.71)$ & $3.61(1.58)$ & $3.77(1.41)_{a}^{a}$ \\
\hline 低 & $5.18(1.42)_{1}$ & $3.48(1.70)_{2}$ & $4.33(1.27)_{b}^{b}$ \\
\hline 平均值 & $4.62(1.40)_{1}$ & $3.69(1.16)_{2}$ & \\
\hline
\end{tabular}

注: 1.不同的角标 $\mathrm{a}$ 和 $\mathrm{b}, 1$ 和 2 表示二者间有显著差异， $p<0.05,1$ 和 2 表示二者之间存在边缘显著差异, $p<0.10$ 2. 各维度的值为：内外维度 1 (外的) 到 7 (内的);稳定性 维度 1（不稳定）到 7（稳定的）;普遍性维度 1（特殊的） 到 7 (普遍的); 可控制性维度 1 (不可控的) 到 7 (可控的)

\section{5. 讨论}

在 4 个原因维度, 内群成员面试结果的主效应显 著。即内群成员的成功比失败被归于更为内在的、稳 定的、普遍的、可控的原因, 内群偏袒归因倾向表现 在原因的 4 个维度，假设 1 得到证实。

在对群际归因偏好最为重要的内外维度 ${ }^{[11]}$ 中, 无 一致性信息条件中表现出内群偏祖归因倾向, 但在其 他的 3 个有一致性信息的条件(一致性不明确、高、低 条件)内群偏袒归因倾向消失。这表明与有一致性信 息时相比无时内群偏祖归因倾向更为显著, 一致性信 息的有无对内群偏袒归因倾向有显著影响。假设 2 得 到证实。其原因可以被解释为在给予一致性信息时对 其进行理性归因的同时, 对内群的自我归类意识变得 不显著导致内群偏袒归因倾向被削弱。

另外, 在可控制性维度, 一致性低、无、不明 确条件中表现出群际归因偏好, 但一致性高时未表 现出内群偏袒归因倾向。即内群成员成功别人也成 功时不比内群成员失败别人也失败时更加归于内群 成员的可控原因。因此, 在一致性信息中, 存在容 易显著表现出内群偏袒归因倾向的情形。

\section{4. 总讨论}

\section{1. 对一致性信息的归因}

在预实验及正式实验中中, 对一致性信息进行理 性归因的结果，一致性低时被归于内的、稳定的、普 遍的、可控的原因; 一致性高时被归于较为外在的、 不稳定的、特殊的、不可控的原因。这些结果支持 $\mathrm{Ma}^{[13,14]}$ 的研究中中关于一致性信息的归因预测。其原 因可以解释为一致性低/高时差异法与契合法分别起 作用分别被归于内/外因,而一致性程度不明确或无一 致性信息时则由于契合法与差异法不起作用其归因 在一致性高引起的外归因与一致性低引起的内归因 之间。

\section{2. 一致性信息对内群偏袒归因的削弱效果}

在预实验中，一致性信息理性归因的同时，客家 人对客家人内群表现出内群偏袒, 而未表现出贬低外 群。因此客家人没有表现出群际归因偏好。在正式实 验中, 无一致性信息时表现出内群偏袒归因倾向; 给 予一致性信息时则未表现出内群偏袒归因倾向。无一 致性信息时比有时内群偏袒归因倾向更为显著, 一致 性信息的有无对内群偏袒归因倾向有显著影响。

从两个实验的结果综合来看, 一致性信息对于内 群偏袒归因倾向在内的群际归因偏好有显著的干扰 作用。其原因可以解释为对于一致性信息进行理性归 因的同时, 产生去类化, 内群偏袒归因倾向及群际归 因偏好受到削弱。群际归因偏好主要有内群认同强度、 群体显著性等影响因素 ${ }^{8,12]}$, 但很少有研究探讨归因 的背景信息及线索对群际归因偏好的影响, 而本研究 的结果表明一致性信息对群际归因偏好具有削弱效 果。

\section{3. 一致性高: 在可控制性维度内群偏袒归因 倾向消失的特殊情形}

在可控制性维度，一致性低、无、不明确条件中 表现出内群偏袒归因倾向，但一致性高时未表现出内 群偏袒归因倾向。因此，和在可控制性维度表现出内 群偏祖归因倾向的一致性低、不明确及无时相比，一 致性高是内群偏袒归因倾向在可控制性维度消失的 特殊情形。

而 $\mathrm{Ma}^{[14]}$ 的结果表明, 在可控制性维度, 一致性 低、不明确及无时利已归因偏好得到证实，但一致性 高时在可控制性维度利已归因偏好消失。因此, 从本 研究与 $\mathrm{Ma}^{[14]}$ 的结果综合来看, 和在可控制性维度表 现出利已归因倾向与内群偏袒归因倾向的一致性低、 不明确及无时相比, 一致性高是利已归因倾向与内群 偏袒归因倾向在可控制性维度消失的特殊情形。 


\section{5. 不足与展望}

虽然本研究通过两个实验证实了给予一致性信 息能减轻群际归因偏好。但至少还存在三个问题。首 先，正式实验由于没有设置外群体而未进行内外群间 的归因比较。而对于群际归因偏好, 有必要进行内外 群间的归因比较。其次, 本研究中无一致性信息时比 有时内群偏祖归因倾向及群际归因偏好表现更为显 著的原因可以解释为在给予一致性信息时对其进行 理性归因, 同时内外群显著性及对内群的自我归类意 识变得不显著。但均未对有无一致性信息时的内外群 显著性及对内群的自我归类意识的差异程度进行直 接测量。第三, 本研究只探讨了一致性信息对群际归 因偏好的削弱效果，而未探讨如一贯性信息等的其他 种类的共变信息对群际归因偏好的削弱效果。另外, 未探讨同时给予两三种类的共变信息组合及如低一 致性/高一贯性/低区别性(被归因于行为者)等的一些 能引起明确理性归因的典型共变信息组合时对群际 归因偏好的削弱效果 ${ }^{[10]}$ 。

\section{6. 结论}

1. 无一致性信息时客家人表现出内群偏袒归因 倾向, 但给予一致性信息时内群偏祖归因倾向消失。 一致性信息的有无对内群偏祖归因倾向有显著影响, 一致性信息对内群偏祖归因倾向有显著的干扰作用。

2. 在可控制性维度, 一致性低、无、不明确条件 中表现出内群偏祖归因倾向, 但一致性高时内群偏祖 归因倾向消失

\section{REFERENCES}

[1] Ariyanto, A., Hornsey, M. J., \& Gallois, C. (2009). Intergroup attribution bias in the context of extreme intergroup conflict. Asian Journal of Social Psychology, 12(4), 293-299.

[2] Bradley, G. W. (1978). Self-serving biases in the attribution process: A reexamination of the fact or fiction question. Journal of Personality and Social Psychology, 36(1), 56-71.

[3] Brewer, M. B., \& Miller, N. (1984). 13 - Beyond the Contact Hypothesis: Theoretical Perspectives on Desegregation. In N. Miller \& M. B. Brewer (Eds.), Groups in Contact: Academic Press.pp. 281-302

[4] De Michele, P., Gansneder, B., \& Solomon, G. (1998). Success and failure attributions of wrestlers: Further evidence of the self-serving bias. Journal of Sport Behavior, 21, 242-255.

[5] Doosje, B., \& Branscombe, N. R. (2003). Attributions for the negative historical actions of a group. European Journal of Social Psychology, $33(2), 235-248$.
[6] Fontaine, G. (1975). Causal attribution in simulated versus real situations: When are people logical, when are they not? Journal of Personality and Social Psychology, 32(6), 1021-1029.

[7] Guerin, B. (1999). Children's intergroup attribution bias for liked and disliked peers. The Journal of Social Psychology, 139(5), 583-589.

[8] Hewstone, M. (1990). The 'ultimate attribution error'? A review of the literature on intergroup causal attribution. European Journal of Social Psychology, 20(4), 311-335.

[9] Islam, M., \& Hewstone, M. (1993). Intergroup Attributions and Affective Consequences in Majority and Minority Groups. Journal of Personality and Social Psychology, 64, 936-950.

[10] Kelley, H. (1967). Attribution Theory in Social Psychology. In (Vol. 14, pp. 192-238).

[11] Ma. Weijun. (2005). A Research Regarding Information Processing Models and Biases in Causal Attribution. Unpublished Doctorial Dissertation, Kobe University, Japan. (in Japanese)

[12] Ma, W. (2009). A Review on Studies of Intergroup Attributional Bias. Psychological Science, 32(4), 908-909,912.

[13] Ma, W. (2010). The Attributional of One Covariation Information on Causal Dimensions, 33(4), 891-895.

[14] Ma, W. (2011). The Influences of Extents' Differences of Covariation Information on Attributions, 34(6), 1469-1475.

[15] Miller, N., Brewer, M., \& Edwards, K. (2010). Cooperative Interaction in Desegregated Settings: A Laboratory Analogue. Journal of Social Issues, 41, 63-79.

[16] Pettigrew, T. (1979). The Ultimate Attribution Error: Extending Allport's Cognitive Analysis of Prejudice. Personality and Social Psychology Bulletin, 5, 461-476.

[17] Stephan, W. G. (1977). Stereotyping: The roles of ingroup-outgroup differences in causal attribution for behavior. The Journal of Social Psychology, 101(2), 255-266.

[18] Tajfel, H., \& Turner, J. C. (2010). An integrative theory of intergroup conflict. In T. Postmes \& N. R. Branscombe (Eds.), Rediscovering social identity. New York, NY: Psychology Press. pp. 173-190.

[19] Taylor, D. M., \& Jaggi, V. (1974). Ethnocentrism and causal attribution in a south Indian context. 
Journal of Cross-Cultural Psychology, 5(2), 162171.

[20] Van Overwalle, F. J., \& Heylighen, F. P. (1995). Relating covariation information to causal dimensions through principles of contrast and invariance. European Journal of Social Psychology, 25(4), 435-455.

[21] Weber, J. G. (1994). The nature of ethnocentric attribution bias: Ingroup protection or enhancement? Journal of Experimental Social Psychology, 30(5), 482-504. 